\title{
TWEAK and its receptor Fn14 in the synovium of patients with rheumatoid arthritis compared to psoriatic arthritis and its response to tumour necrosis factor blockade
}

\author{
A W R van Kuijk, ${ }^{1}$ C A Wijbrandts, ${ }^{1}$ M Vinkenoog, ${ }^{1}$ T S Zheng, ${ }^{2}$ K A Reedquist, ${ }^{1}$ P P Tak
}

${ }^{1}$ Division of Clinical Immunology and Rheumatology, Academic Medical Center/University of Amsterdam, The Netherlands; ${ }^{2}$ Biogen Idec, Cambridge, Massachusetts, USA

Correspondence to:

P P Tak, Division of Clinical Immunology and Rheumatology, F4-218, Academic Medical Center/University of Amsterdam, Meibergdreef 9, NL-1105 AZ

Amsterdam, The Netherlands; p.p.tak@amc.uva.nl

The first two authors contributed equally to this manuscript.

Accepted 16 December 2008 Published Online First

15 January 2009

\section{ABSTRACT}

Objective: To investigate the expression of tumour necrosis factor (TNF)-like weak inducer of apoptosis (TWEAK) and its receptor fibroblast growth factor inducible 14 (Fn14) in the inflamed synovium of patients with arthritis, as TWEAK blockade has been observed to have a beneficial effect in an animal model of rheumatoid arthritis (RA).

Methods: Synovial tissue (ST) biopsies were obtained from 6 early, methotrexate-naive patients with RA as well as 13 patients with RA and 16 patients with psoriatic arthritis (PsA) who were matched for treatment and disease duration. Serial ST samples were obtained from a separate cohort of 13 patients with RA before and after infliximab treatment. TWEAK and Fn14 expression was evaluated by immunohistochemistry and digital image analysis.

Results: TWEAK and Fn14 were clearly expressed in ST of patients with RA and PSA. TWEAK expression was significantly higher in $\mathrm{RA}$ (sub))lining samples compared to PsA ( $p=0.005$ and $p=0.014$, respectively), but Fn14 expression was comparable. Double immunofluorescence showed TWEAK and Fn14 expression on fibroblast-like synoviocytes and macrophages, but not $T$ cells. Of interest, persistent TWEAK and Fn14 expression was found after anti-TNF therapy.

Conclusions: TWEAK and Fn14 are abundantly expressed in the inflamed synovium of patients with RA and PsA. This raises the possibility that blocking TWEAK/ Fn14 signalling could be of therapeutic benefit in inflammatory arthritis.

Tumour necrosis factor (TNF)-like weak inducer of apoptosis (TWEAK) is a TNF ligand superfamily member that mediates pleiotropic effects on a variety of cells via its receptor, fibroblast growth factor inducible 14 (Fn14). The TWEAK/Fn14 pathway appears to have a physiological role in the regulation of tissue repair after injury, when Fn14 expression is highly induced. ${ }^{1}$ Fn14 can be expressed by many cell types, including epithelial, mesenchymal and endothelial cells, and progenitor cells of the mesenchymal lineage. ${ }^{3}$ Activating Fn14 can have a number of effects, depending on cell type and context, including proangiogenic effects and induction of fibroblast-like synoviocytes (FLS) to produce proinflammatory cytokines and chemokines, such as interleukin (IL)6, IL8 and Regulated on Activation, Normal T Expressed and Secreted (RANTES). ${ }^{3}{ }^{4}$ TWEAK may also promote bone and cartilage destruction through inhibition of chondrogenesis and osteogenesis and promotion of osteoclastogenesis. ${ }^{2}$
These data raise the possibility that TWEAK may contribute to chronic synovitis, a notion supported by the observation that TWEAK expression is dramatically elevated in the murine collagen-induced arthritis (CIA) model of arthritis. Blocking TWEAK signalling reduced the severity of arthritis in this model, and diminished synovial inflammation, vascularity, and cartilage and bone destruction. ${ }^{2}{ }^{5}$ However, very little is known about the role of the TWEAK/Fn14 pathway in human inflammatory arthritis. To address this issue, we examined the expression of TWEAK and Fn14 in synovial tissue (ST) of patients with rheumatoid arthritis (RA) and psoriatic arthritis (PsA), in newly diagnosed, previously untreated patients with RA, and in patients with RA on methotrexate before and after treatment with infliximab.

\section{PATIENTS AND METHODS}

\section{Patients and synovial tissue acquisition}

For a comparative analysis of TWEAK and Fn14 expression, ST biopsies were obtained by arthroscopy from 13 patients with RA and 16 patients with PsA with clinically active arthritis. ST biopsies from a second cohort of 13 patients with RA on methotrexate (MTX) therapy were obtained from the same joint before and 4 weeks after the initiation of infliximab (IFX) therapy (IFX $3 \mathrm{mg}$ / $\mathrm{kg}$, administered intravenously at baseline and 2 weeks later). ${ }^{6}$ Additionally, ST biopsies were obtained from 6 patients newly diagnosed as having RA who had not been treated with any disease-modifying antirheumatic drug (DMARD). All patients with RA met the 1987 revised criteria of the American College of Rheumatology for the diagnosis of RA. ${ }^{7}$ All patients with PsA fulfilled the CASPAR (for "ClASsification of Psoriatic ARthritis") group criteria, ${ }^{8}$ and had active joint and skin disease at the time of arthroscopy. ST biopsies were obtained by arthroscopy from an actively inflamed knee, ankle or wrist joint under local anaesthesia. ${ }^{9}$ Biopsies were obtained from six or more sites in each joint. ST biopsies were immediately embedded in TissueTek OCT (Miles Diagnostics, Elkhart, Indiana, USA), snap frozen in liquid nitrogen and stored at $-80^{\circ} \mathrm{C}$, as previously described in detail. ${ }^{10}$ All patients provided their written informed consent and patient clinical and demographic data were obtained before arthroscopy. This study was approved by the local medical ethics committee. 
Table 1 Demographic data of the first cohort of patients with psoriatic arthritis (PsA) and rheumatoid arthritis (RA)

\begin{tabular}{lcc}
\hline & PsA (n= 16) & RA ( $\mathbf{n = 1 3 )}$ \\
\hline Female, no (\%) & $6(38 \%)$ & $6(46 \%)$ \\
Age in years, mean (range) & $46.5(22-64)$ & $52.6(38-69)$ \\
Disease duration in months, mean (range) & $120(39-284)$ & $137(10-278)$ \\
RF and/or anti-CCP positive, no (\%) & $2(13 \%)$ & $13(100 \%)$ \\
Erosions, no (\%) of patients & $8(50 \%)$ & $11(88 \%)$ \\
ESR mm/h, median (IOR) & $21.5(7.3-32.3)$ & $28.0(9.5-47.5)$ \\
CRP mg/litre, median (IQR) & $10.4(2.5-23.7)$ & $7.0(3.5-35.5)$ \\
Methotrexate dose (mg), mean (SD) & $16.7(7.2)$ & $20.0(4.3)$ \\
\hline
\end{tabular}

Patients with RA were more often RF and/or anti-CCP positive than patients with PsA. No other significant differences in the clinical data between patients with RA and PsA were detectable.

CCP, cyclic citrullinated peptide; CRP, C-reactive protein; ESR, erythrocyte sedimentation rate; IQR, interquartile range (25th-75th percentile); RF, rheumatoid factor.

\section{Immunohistochemistry and digital image analysis}

ST samples were cut with a cryostat $(5 \mu \mathrm{m})$, fixed with acetone, and endogenous peroxidase activity was blocked with $0.3 \%$ hydrogen peroxide. Specific monoclonal antibodies were used to detect TWEAK (clone P3H8) and Fn14 (clone P2D3) (both from
Biogen Idec, Cambridge, Massachusetts, USA). Bound antibody was detected with a three-step immunoperoxidase method using a biotinylated tyramine amplification method. ${ }^{11}$ Stained sections were then randomly analysed using digital image analysis (DIA), as previously described in detail. ${ }^{12}$ Expression of TWEAK and Fn14 is presented as integrated optical density (IOD) $/ \mathrm{mm}^{2}$, an arbitrary unit representing the amount of staining per $\mathrm{mm}^{2}{ }^{13}$

\section{Double immunofluorescence}

TWEAK and Fn14 expression in distinct synovial cell populations was assessed by double immunofluorescence in 15 randomly selected patients ( 8 with PsA and 7 with RA). Sections were incubated overnight at $4^{\circ} \mathrm{C}$ with primary monoclonal antibodies against TWEAK and Fn14, and then labelled with streptavidin-ALEXA 594 (Invitrogen/Molecular Probes, Breda, The Netherlands) for $30 \mathrm{~min}$. Next, sections were blocked with 10\% normal mouse serum, incubated for 30 min with fluorescein isothiocyanate (FITC)-labelled markers for CD55 (clone BRIC 110, Sanquin, Amsterdam, The Netherlands), CD3 (clone SK7, Becton Dickinson, San Jose, California, USA) and CD68 (clone KP1, Dako, Glostrop,

\section{RA}
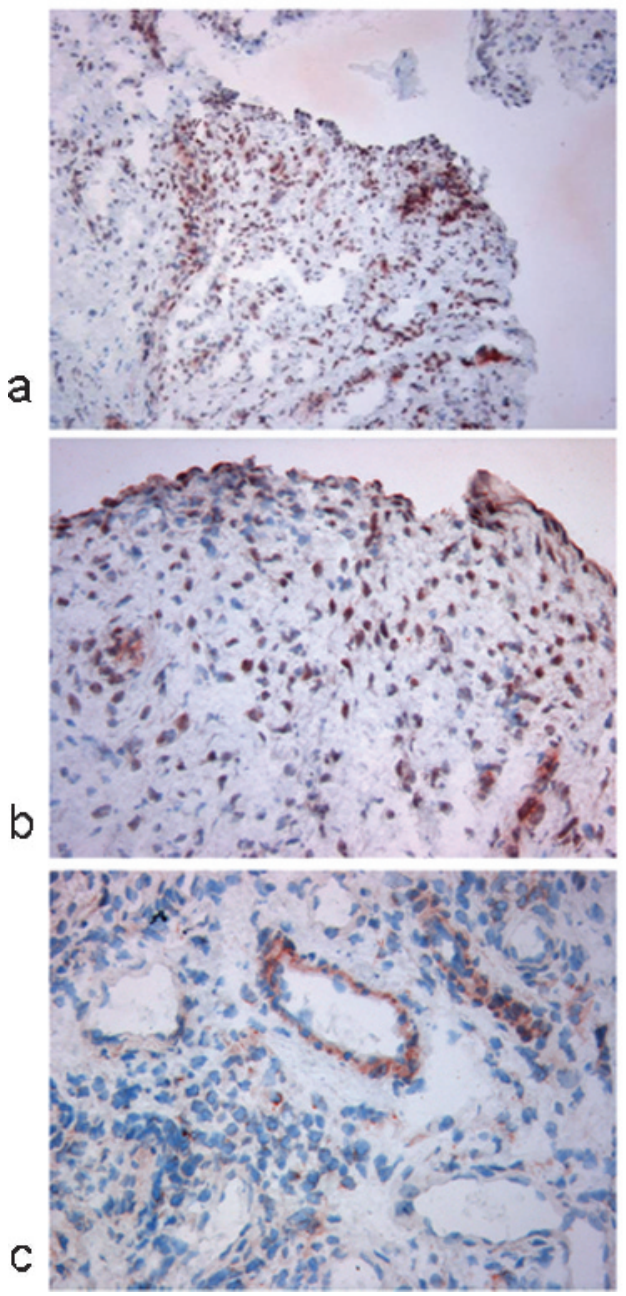

PsA
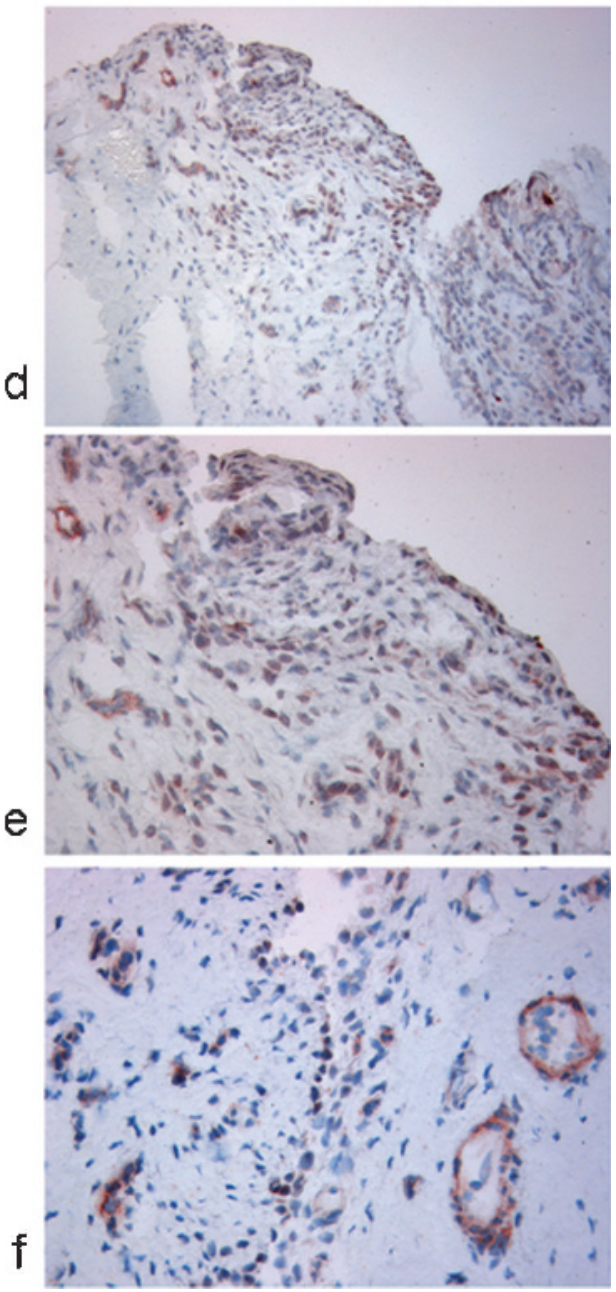

Figure 1 Representative photographs showing tumour necrosis factor (TNF)-like weak inducer of apoptosis (TWEAK) expression (reddish-brown staining) in rheumatoid arthritis (RA) synovial tissue (ST) (a-c) and psoriatic arthritis (PSA) ST (d-f). Overview of RA ST (a) (original magnification $100 \times$ ), and close up of the intimal lining layer (b) and the synovial sublining with (peri)vascular staining (c) (magnification $200 \times$ ); overview of PsA ST (d), and close up of the intimal lining layer (e) and the synovial sublining with (peri)vascular staining (f). 
RA

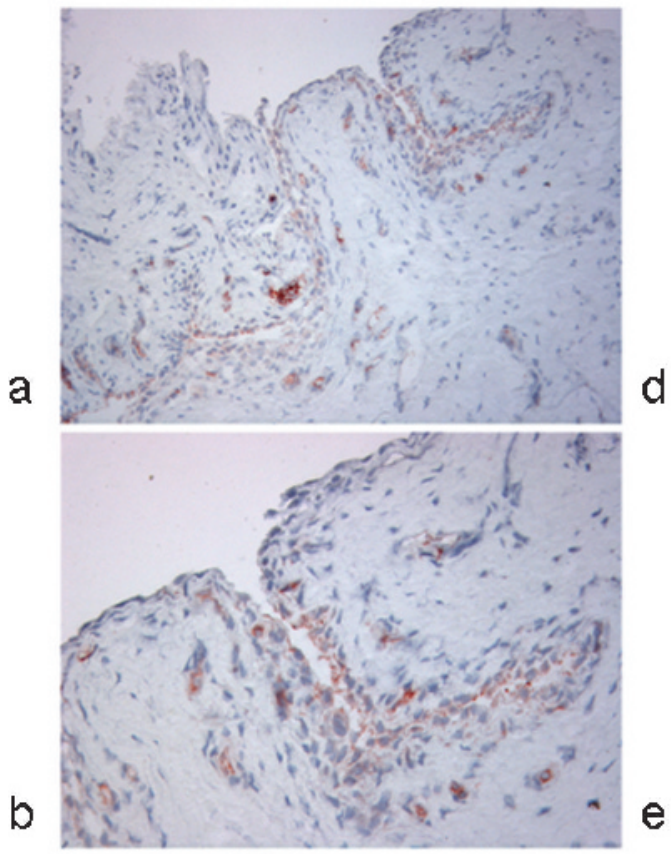

C

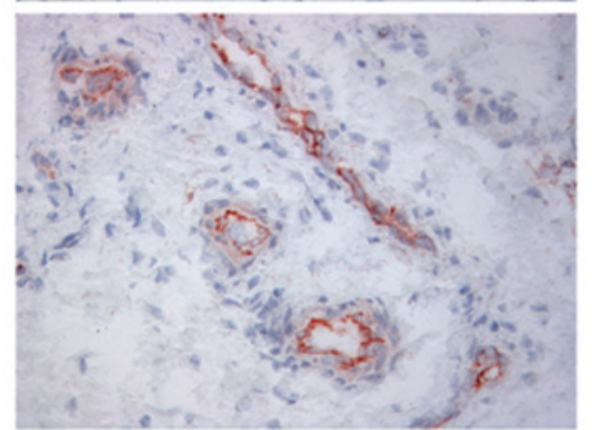

PsA
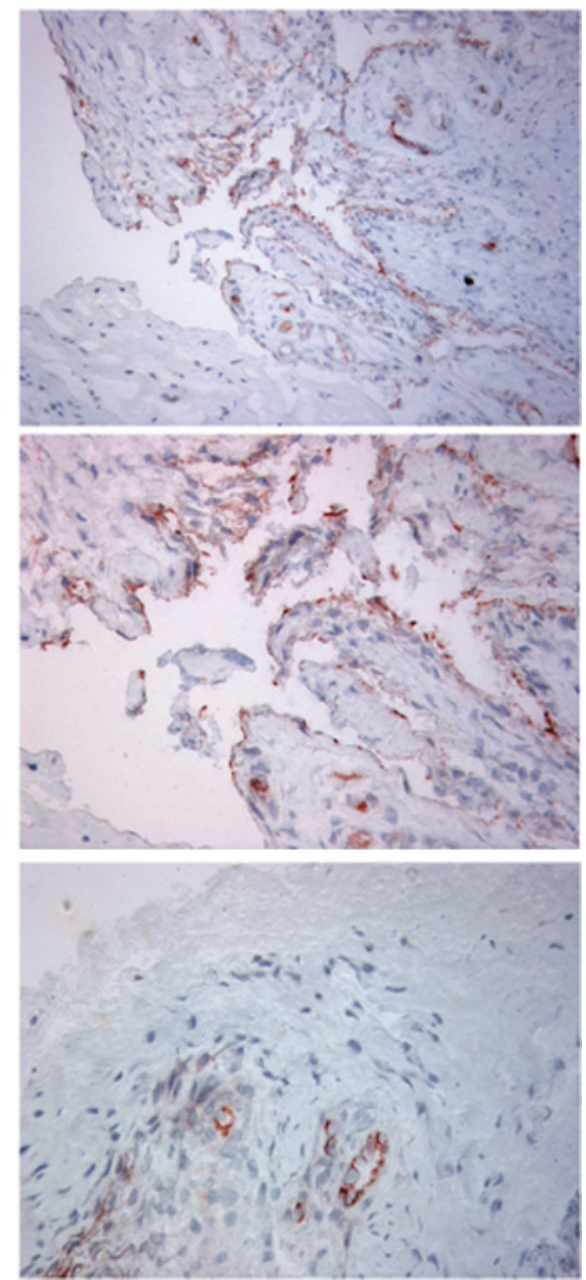

Figure 2 Representative photographs showing tumour necrosis factor (TNF)-like weak inducer of apoptosis (TWEAK) receptor fibroblast growth factor inducible 14 (Fn14) expression (reddish-brown staining) in rheumatoid arthritis (RA) synovial tissue (ST) (a-c) and psoriatic arthritis (PsA) synovial tissue (d-f). Overview of RA ST (a) (original magnification $100 \times$ ), and close up of the intimal lining layer (b) and the synovial sublining (c) (magnification $200 \times$ ); overview of PSA ST (d), and close up of the intimal lining layer (e) and the synovial sublining (f).

Denmark), covered with VectaShield mounting medium (H-1200, Vector laboratories, Burlingame, California, USA), and analysed with a wide-field upright microscope (Leica DMRA, Wetzlar, Germany) coupled to a CCD camera and Image-Pro Plus software (Media Cybernetics, Dutch Vision Components, Breda, The Netherlands). Coexpression of CD68, CD55 and CD3 with TWEAK and Fn14 was determined by the manual counting of positive cells by two independent blinded observers (Tom Smeets and Dion Groot, Academic Medical Center/University of Amsterdam, Amsterdam, The Netherlands).

\section{Statistical analysis}

To compare the differences in expression of TWEAK and Fn14 between groups the Mann-Whitney $U$ test for non-parametric data was used. Correlations were assessed using the Spearman rank correlation coefficient. Serial biopsies were evaluated using the Wilcoxon signed rank test. A $p$ value of $<0.05$ was considered statistically significant.

\section{RESULTS}

Table 1 shows the patient characteristics of the first patient cohort (13 patients with RA and 16 with PsA) who were used for cross sectional comparison. All these patients were treated with methotrexate; there was a trend towards slightly higher dosages in RA, but this difference was not statistically significant. In both groups one patient was also treated with prednisolone $10 \mathrm{mg}$ daily. Most patients had longstanding disease and displayed comparable erythrocyte sedimentation rate (ESR) and C-reactive protein (CRP) levels, indicating similar degrees of inflammatory activity.

In RA and PsA ST, strong expression of TWEAK was observed in the intimal lining layer and the synovial sublining, including perivascular regions in a subset of blood vessels (fig 1). Expression of TWEAK was higher in RA than in PsA in the intimal lining layer (median and interquartile range (IOR)), respectively 45305 (21 410-63 456) versus $14381 \quad(934-20002) \quad \mathrm{IOD} / \mathrm{mm}^{2}$ $(p=0.005)$ and the synovial sublining 44941 (30 687-106 183) versus $8022(2484-70439) \mathrm{IOD} / \mathrm{mm}^{2}(p=0.014)$. Fn14 was expressed in the intimal lining layer and the perivascular regions of the synovial sublining (fig 2). Fn14 expression was not significantly different between RA and PsA. Immunofluorescent double staining revealed frequent TWEAK (65\%) and Fn14 (46\%) expression in CD55+ RA FLS. CD68+ macrophages also frequently expressed TWEAK (46\%) and Fn14 (20\%). In contrast, CD3+ T cells hardly expressed any TWEAK or Fn14. 
A second cohort of 13 patients with RA underwent arthroscopy before and 4 weeks after initiation of infliximab infusions. Patients had longstanding disease (mean disease duration 127 months, range 10-311), all were treated with MTX (mean dose $15 \mathrm{mg} /$ week, range 5-30) and 10/13 with prednisolone (mean dose $9 \mathrm{mg} /$ day, range 2.5-15). The 28-joint Disease Activity Score (DAS28) was significantly reduced following 4 weeks of infliximab therapy: DAS28 (median and IOR) at baseline, 6.25 (5.57-7.04); after 4 weeks, 4.48 (3.825.98) $(p<0.01)$. However, there was persistent TWEAK expression after TNF blockade without any significant changes in ST TWEAK or Fn14 expression following infliximab therapy.

To rule out the possibility that the expression of TWEAK and Fn14 had been influenced considerably by disease duration or DMARD treatment in the above mentioned cohorts, ST samples from a third cohort of 6 recently diagnosed patients with RA who had not yet been treated with DMARDs was studied and compared to the 13 patients with RA from the second cohort. The expression of TWEAK and Fn14 was comparable between this group with early disease and the abovementioned group with longstanding disease before start of infliximab therapy, indicating that TWEAK and its receptor are expressed in early RA.

\section{DISCUSSION}

TWEAK and Fn14 are both abundantly expressed in the inflamed synovium of patients with (early) RA and PsA, where they may promote synovial inflammation and joint destruction. Of importance, there is persistent expression of TWEAK and its receptor after TNF blockade. TWEAK expression in the intimal lining layer and synovial sublining is significantly higher in RA compared to PsA, while Fn14 expression is similar. TWEAK and Fn14 are mainly expressed by FLS and, to a lesser extent, macrophages, suggesting that both effector cells may participate in TWEAK responses in patients with arthritis.

The TWEAK/Fn14 pathway appears to have a physiological role in the regulation of tissue repair after injury. TWEAK may initially be produced by tissue cells, and by invading inflammatory cells such as monocytes. The TWEAK-Fn14 pathway might then drive chemokine and cytokine production, resulting in additional infiltration of proinflammatory cells, and facilitate angiogenesis and the proliferation of progenitor cells needed for tissue repair. Chronic expression of TWEAK and Fn14 in inflammatory arthritis may subvert processes beneficial in acute tissue repair to pathogenic contributions. The expression of TWEAK and Fn14 in chronic synovitis could contribute to persistent inflammation and progressive destruction of the joint tissue by the production of chemokines, cytokines and MMPs, and promotion of cell proliferation and angiogenesis. Because the TWEAK/Fn14 pathway does not appear to be involved in the regulation of adaptive immunity, it has been suggested that blocking TWEAK/Fn14 activation would potentially have a favourable safety profile. Further studies are needed to identify the contributions of TWEAK/Fn14 to inflammatory arthritis, and the therapeutic potential of targeting this pathway.

Acknowledgements: The authors wish to thank Dr Tom JM Smeets and Dion Groot for their work on the immunohistochemical staining and immunofluorescent double staining.

Funding: This study was supported by Biogen Idec, Cambridge, Massachusetts, USA. This research was also supported by the European Community's FP6 funding ("Autocure"). This publication reflects only the author's views; the European Community is not liable for any use that may be made of the information herein.

Competing interests: TSZ is an employee of Biogen Idec, Cambridge,

Massachusetts, USA.

Ethics approval: Ethics approval was granted by the Medical Ethics Committee of the Academic Medical Center/University of Amsterdam, Amsterdam, The Netherlands.

Provenance and peer review: Not commissioned; externally peer reviewed.

\section{REFERENCES}

1. Burkly LC, Michaelson JS, Hahm K, et al. TWEAKing tissue remodeling by a multifunctional cytokine: role of TWEAK/Fn14 pathway in health and disease. Cytokine 2007; 40:1-16.

2. Perper SJ, Browning B, Burkly LC, et al. TWEAK is a novel arthritogenic mediator. J Immunol 2006;177:2610-20.

3. Wiley SR, Winkles JA. TWEAK, a member of the TNF superfamily, is a multifunctional cytokine that binds the TweakR/Fn14 receptor. Cytokine Growth Factor Rev 2003;14:241-9.

4. Chicheportiche $\mathbf{Y}$, Chicheportiche R, Sizing I, et al. Proinflammatory activity of TWEAK on human dermal fibroblasts and synoviocytes: blocking and enhancing effects of anti-TWEAK monoclonal antibodies. Arthritis Res 2002;4:126-33.

5. Kamata K, Kamijo S, Nakajima A, et al. Involvement of TNF-like weak inducer of apoptosis in the pathogenesis of collagen-induced arthritis. J Immunol 2006;177:6433-9.

6. Smeets TJ, Kraan MC, van Loon ME, et al. Tumor necrosis factor alpha blockade reduces the synovial cell infiltrate early after initiation of treatment, but apparently not by induction of apoptosis in synovial tissue. Arthritis Rheum 2003:48:2155-62.

7. Arnett FC, Edworthy SM, Bloch DA, et al. The American Rheumatism Association 1987 revised criteria for the classification of rheumatoid arthritis. Arthritis Rheum 1988;31:315-24.

8. Taylor W, Gladman D, Helliwell $P$, et al. Classification criteria for psoriatic arthritis: development of new criteria from a large international study. Arthritis Rheum 2006;54:2665-73.

9. Kraan MC, Reece RJ, Smeets TJ, et al. Comparison of synovial tissues from the knee joints and the small joints of rheumatoid arthritis patients: implications for pathogenesis and evaluation of treatment. Arthritis Rheum 2002;46:2034-8.

10. Gerlag D, Tak PP. Synovial biopsy. Best Pract Res Clin Rheumatol 2005;19:387-400.

11. Smeets TJ, Barg EC, Kraan MC, et al. Analysis of the cell infiltrate and expression of proinflammatory cytokines and matrix metalloproteinases in arthroscopic synovial biopsies: comparison with synovial samples from patients with end stage, destructive rheumatoid arthritis. Ann Rheum Dis 2003;62:635-8.

12. Haringman JJ, Vinkenoog $\mathbf{M}$, Gerlag DM, et al. Reliability of computerized image analysis for the evaluation of serial synovial biopsies in randomized controlled trials in rheumatoid arthritis. Arthritis Res Ther 2005;7:R862-7.

13. van der Hall PO, Kraan MC, Tak PP. Quantitative image analysis of synovial tissue Methods Mol Med 2007;135:121-43. 